\title{
Vectors and Integration in Gene Therapy: Statistical Considerations
}

\author{
Alessandro Ambrosi ${ }^{1}$, Clelia Di Serio ${ }^{1 *}$ \\ ${ }^{1}$ University Centre of Statistics for Biomedical Sciences (CUSSB), \\ Università Vita-Salute San Raffaele, Via Olgettina, 58 - 20132 Milano
}

*Corresponding author: Clelia Di Serio, University Centre of Statistics for Biomedical Sciences (CUSSB), Università Vita-Salute San Raffaele, Via Olgettina, 58 -20132 Milano, E-mail: diserio.clelia@ hsr.it

Received February 23, 2009; Accepted February 25, 2009; Published February 27, 2009

Citation: Alessandro A, Di Serio C (2009) Vectors and Integration in Gene Therapy: Statistical Considerations. J Comput Sci Syst Biol 2: 117-123. doi:10.4172/jcsb.1000023

Copyright: (C) 2009 Alessandro A, et al. This is an open-access article distributed under the terms of the Creative Commons Attribution License, which permits unrestricted use, distribution, and reproduction in any medium, provided the original author and source are credited.

\section{Abstract}

In gene therapy the integration process of the viral DNA genome into the host cell genome is a necessary step for virus integration. Just few years ago, retrovirus integration was believed to be random and the chance of accidentally activating a gene was considered remote. It has been seen that this process is not random and different viruses may show different preferences to integrate in some specific areas of the genome. Tumorigensis associated to some studies in gene therapy is suspected to be caused by insertion process. Depending on whether the provirus integrates into or in the vicinity of genes (Transcription Start Sites, TSS), normal trascription can be enhanced or disrupted thus inducing oncogenic mutations. This is called "insertional mutagenesis". Investigating whether an area over the genome could be favoured by retrovirus integration is a crucial aspect in gene therapy. These area are called "Common Integration Sites"(CIS)or "hotspots". In the paper we stressed the importance of developing statistical procedures leading to a unique definition of CIS rather than a "problem related" definition. We here propose some statistical solutions for the search of hotspots based on the "Peaksheight distribution", which account within the null hypothesis for the possible non-random behaviour of the integrations.

\section{Background}

Gene therapy is a form of molecular medicine which treats genetic diseases by replacing a defective gene, responsible for the pathology, with a functional one. The basic principle is to introduce a piece of genetic material into cells via a virus which represents the vector for gene therapy. The virus integrates with the cell DNA and thus delivers the genetic material into the cell nucleus. This process is called integration and may alter the host cell's DNA. Recent studies based on cellular and animal models (Bushman, 2005) reported empirical evidence of preference for certain retroviral vectors, i.e. those deriving from Moloney Murine Leukemia Virus (MLV), to integrate near the start of transcriptional units, whereas others (like Simian Immunodefi- ciency Virus (SIV)- and Human Immunodeficiency Virus (HIV)-based vectors) did not show the same tendency. The mutation may alter the expression of genes in the vicinity of the insertion or, when inserted within a gene, alter the gene product. When the affected gene is a cancer gene (either a proto-oncogene or a tumor suppressor gene), activation of the proto-oncogene or inactivation of the tumor-suppressor gene can cause uncontrolled proliferation (cell division) of cells. Eventually this may give rise to tumors. These cancer-causing insertions are referred to as insertional mutagenesis or oncogenic integration. A tumor could develop when an accumulation of oncogenic insertions causes uncontrolled proliferation of a cell. This has been seen both in 
animal as well as human models. The related problem of safety of a vector is a major hurdle (Montini et al., 2006). It has been observed that in retroviral integration different vectors show distinct target site preferences, thus finding a unique statistical criteria to detect accumulation of integration is a fundamental tool within the debate on safety of a vector. (Recchia et al., 2006, Cassani et al., 2006). Some approaches provided statistical and mathematical modelling to to test the hypothesis of randomness (Abel et al., 2007; Ambrosi et al., 2008). Moreover in the recent literature (Cattoglio et al., 2007) it has been proved that analysis of MLV integration patterns in natural or experimentally induced leukemias/lymphomas showed the existence of insertion sites recurrently associated with a malignant phenotype. These "common insertion sites" (CIS), also called "hotspots" which include proto-oncogenes or other genes associated with cell growth and proliferation, may present when activated a causal relationship with the establishment and/or progression of cancer. The definition of hotspot CIS is however not unique and crucially "problem related". A first model to define a hotspot CIS has been provided by Suzuki et al., (2002) and compares the mapped locations of the proviruses in the isolated tumors to randomly generated integrations from 100,000 Monte Carlo trials. This was done to determine cutoffs for defining when two or more integrations in close proximity were significant enough to assume that it didn't happen by chance. Basically the cutoffs were within $30 \mathrm{~kb}$ for 2 integrations, $50 \mathrm{~kb}$ for 3 insertions or $100 \mathrm{~kb}$ for 4 integrations. In terms of the null hypothesis for hotspot CIS analysis this is problematic. Definition of hotspots is based on a comparison to a random set, but there is a clear preference in integration that should be taken into account in the null hypothesis.

Wu et al., (2005) showed that pre-established role in cancer is not sufficient support for the efficacy of the Suzuki CIS technique. For instance expression level in MLV inte gration may also play a role since MLV integrations are biased towards genes with higher expression levels. A first interesting way to account for this non-randomness in the null hypothesis is the Wu et al., model which all ows $75 \%$ of the integrations to occur randomly and $25 \%$ to integrate in a Poisson distribution T5 $\mathrm{kb}$ around the transcriptional start site.

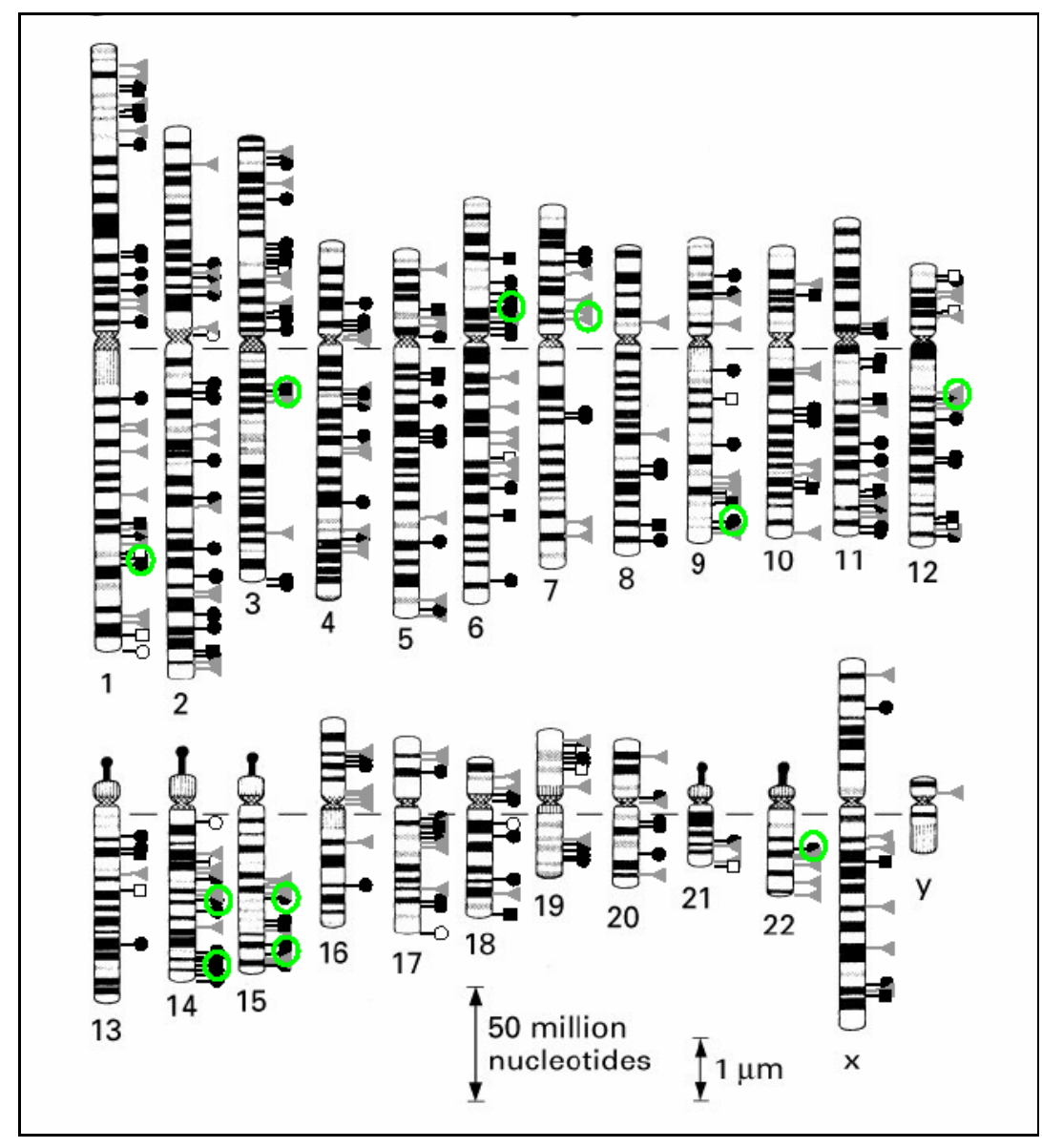

Figure 1: Integration site analysis in T cells. Green circles denote high integration density areas. 
This introduction was aimed at highlighting how statistics and probability must play a fundamental role in establishing a criteria to detect "accumulation" sites and preferences of integration for a better understanding of "how safe" is a vector in gene therapy. To do this we address the following question: how can we distinguish the preferences of viruses to integrate close to TSS from their "accumulation" due to some other reason (for instance to the presence of some particular gene).

\section{Data and Experimental Design}

In this paper we analyzed data derived from retroviral transduction in T cells from leukemic patients treated with allogeneic stem cell transplantation and donor lymphocytes genetically modified with a suicide gene (HSV-TK). Retroviral vectors integrate preferentially within or near transcribed regions of the genome, with a preference for sequences around promoters and for genes active in T cells at the time of transduction. For details on the whole data set see Ambrosi et al., (2008). The following information are reported:

- nucleotide (integration position)

- chromosomes
- integration distance from the TSS of genes in a window of $100 \mathrm{~kb}$

- expression data for genes involved in the integration (hotspots and all)

- gene density in $1 \mathrm{Mb}$ neighbourhood

Figure 1 provides an example of Common Integration Site (CIS) which are classified on the criteria of two integrations occurring in a $100 \mathrm{~kb}$ window. These can be visualized on all chromosomes by high concentration of hit in a small genomic area.

The observed distribution of integration distances from TSS of the nearest gene are provided in Figure 2. This distribution seems consistent with the new findings in gene therapy literature (among other Ambrosi et al., 2008) that TSS sort of "attracts" integrations.

In this paper we provide some statistical proposals to investigate the real distributional "nature" of a hotspot. As mentioned above we want to address the following question: does the integrations distribution reflects a virus natural attitude to integrate in some genetic areas (like TSS) or are rather some genetic areas that do attract

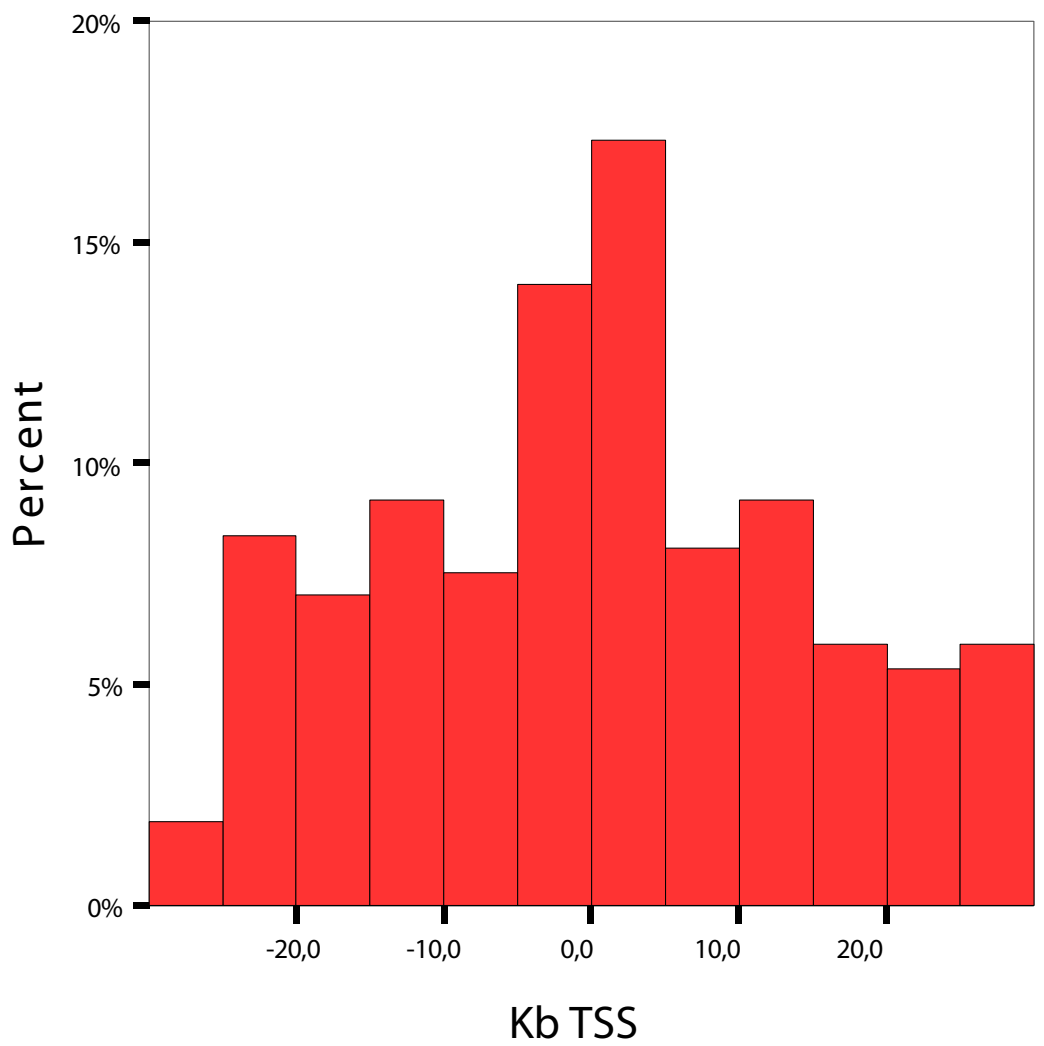

Figure 2: Integration distances distribution from transcription start site of the nearest gene. 

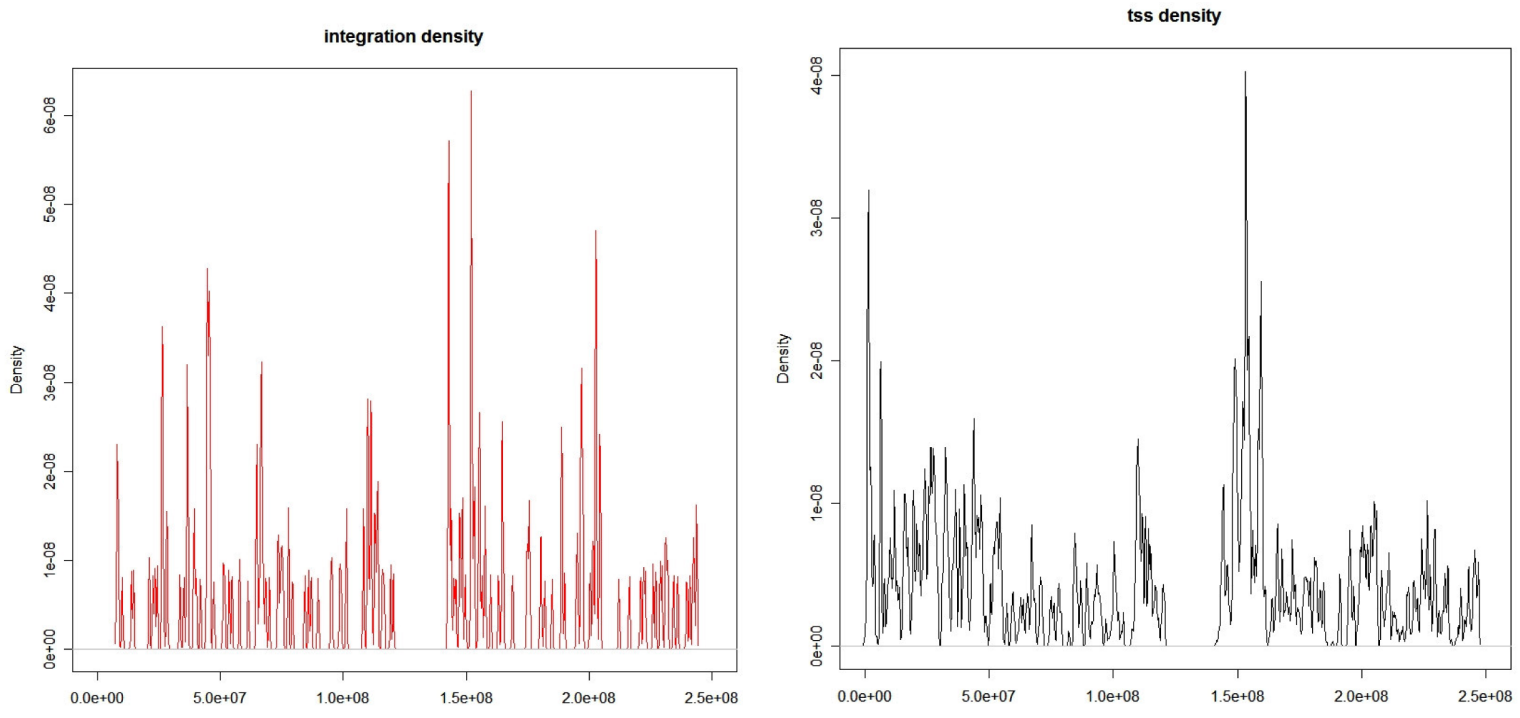

Figure 3: Comparison between the integration density distribution and TSS density distribution.

integrations due to their functional characteristics?

To explore this idea we compare the integration density distribution with the TSS density distribution (which reflects the gene density) like in Figure 3 (referred to the first chromosome). Since the focus of this paper is on the statistical procedure we illustrate our analysis with a small sample of integrations on chromosome 1 only.

It can easily be observed that the two distributions show similarities. This is a natural consequence of the fact that this virus integrates preferably close to the TSS and thus more frequently in high gene density areas. We next focus on those areas that attract insertions of the retrovirus even when no high gene density is revealed.

\section{Statistical Procedure: the Peaks-Hight (P-H) Method}

A natural way to provide a statistical approach for the identification of CIS in distributional terms is a kernel estimation procedure (Ridder et al., 2006) to find the regions in the genome that show a significant increase in insertion density.

For any position over the genome, an estimate of the number of insertions is obtained by summing all the kernel functions. (rectangular, Barlett-Epanechnikov, etc.).

Actually, the basic idea is to model non parametrically the probability that an observation $\mathrm{x}$ will fall into a certain region, that is $F=\int f(x) d x$ with $F$ a smoothed (or aver- aged) version of the density function $\mathrm{f}(\mathrm{x})$. The kernel density estimator $f_{b}(x)$ for the estimation of the density value $f(x)$ at target point $\mathrm{x}$ is a local average smoother that, for random variable $x_{i}$ in a prediction space calculate an average of the observations in a neighbourhood of the target point:

$$
f_{b}(x)=\frac{1}{n b} \sum_{i=1}^{n} k\left(\frac{x_{i}-x}{b}\right)
$$

where $k(\cdot)$ denotes the Kernel function and $b$ is the bandwidth parameter which determines how large a neighbourhood of the target point is. A large bandwidth generates a smoother curve, while a small bandwidth generates a wigglier curve, thus the choice of $\mathrm{b}$ being fundamental and much more important than the choice of kernel (Hastie and Tibshirani, 1990). We use here a standard Gaussian kernel.

Analys is is based on discrete data points indicating the integration position. We are trying to establish how unusual is the spatial patterning of these points. By turning the discrete points into a continuous surface using Kernel estimation, the data can then be explored. In particular we focus on the maximum of the observed integration distribution estimated with by means of a Gaussian kernel. This is now our new random variable, $X_{\mathrm{i}}$ indicating integration peaks height $(\mathrm{P}-\mathrm{H})$.

In the same estimation context we set the null hypothesis, $\mathrm{H}_{0}$ : "integrations occur randomly over the genome" 


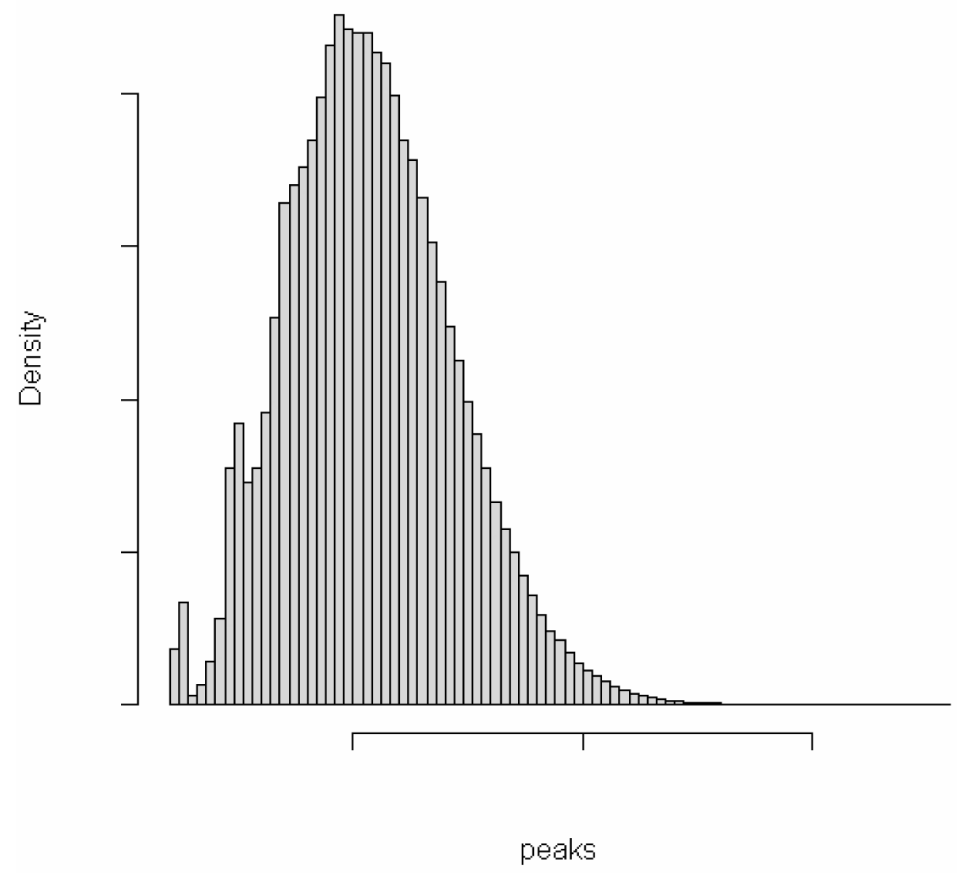

Figure 4: Theoretical peaks distribution under the null hypothesis for Chromosome 1.

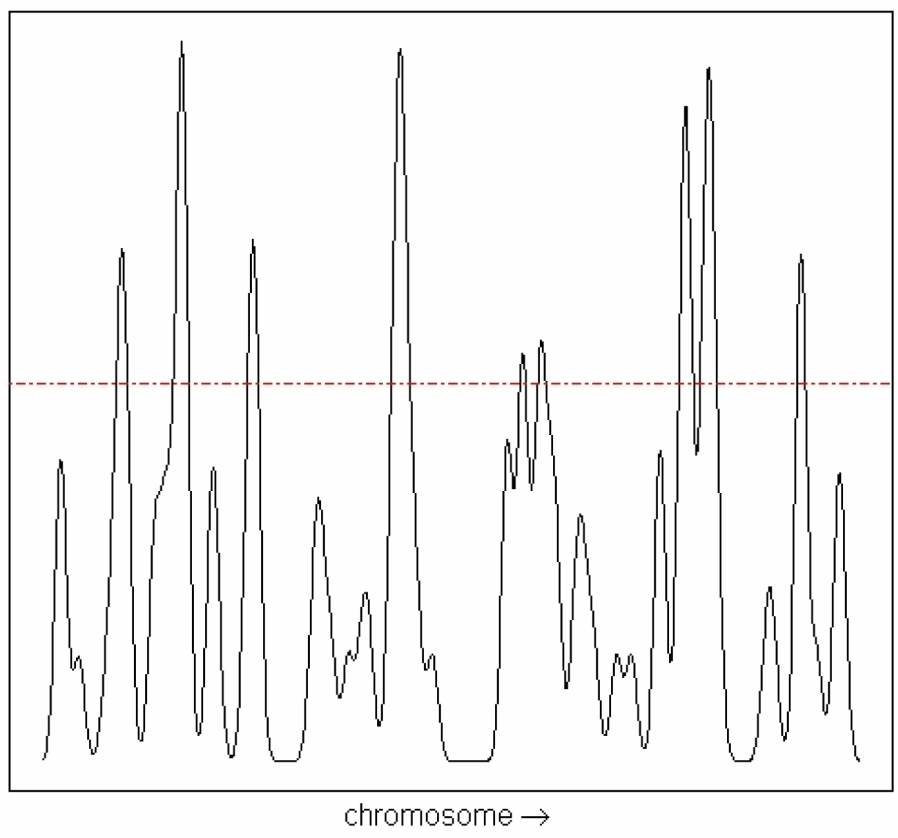

Figure 5: Observed peaks-height distribution. Red line shows the significative peaks with alpha $=0.05$.

(theoretical peaks distribution, Figure 4) to account for natural integration preferences. To build this in terms of peaks we compute the maximum over 1400 integrations which are resampled from a uniform distribution 50000 times via Monte Carlo method.

p-values are then computed for each estimated $\mathrm{P}-\mathrm{H}$ value from the theoretical peaks height distribution (Figure 5).
Correction for multiple comparisons is then applied, and significant $\mathrm{P}-\mathrm{H}$ values are extracted. The neighbourhood of these identifies the "P-H hotspot". In this contribution we present results on Chromosome 1 only. Results reported in Table 1 lead to identify nucleotide positions where a "real" hotspot occurs (that is based on $\mathrm{P}-\mathrm{H}$ based definition of Hotspot). 7 hotspots can be identified after $p$-value corrections for multiple testing. This is a first step that could deal 


\begin{tabular}{|c|c|c|c|c|c|}
\hline $\mathbf{N}$ & where & peak & $p$-value & p.BY & p.Holm \\
\hline 1 & 8312086 & $6.849649 \mathrm{e}-09$ & $2.038982 \mathrm{e}-01$ & 1.0000000000 & $1.000000 \mathrm{e}+00$ \\
\hline 2 & 26779431 & $1.161189 \mathrm{e}-08$ & $1.542289 \mathrm{e}-03$ & 0.0196776871 & $2.467662 \mathrm{e}-02$ \\
\hline 3 & 44754312 & $1.634523 \mathrm{e}-08$ & $4.252635 \mathrm{e}-06$ & 0.0001627750 & $8.930533 e-05$ \\
\hline 4 & 54357331 & $6.687772 \mathrm{e}-09$ & $2.278222 \mathrm{e}-01$ & 1.0000000000 & $1.000000 \mathrm{e}+00$ \\
\hline 5 & 66176432 & $1.183405 \mathrm{e}-08$ & $1.190738 \mathrm{e}-03$ & 0.0182307983 & $2.024254 \mathrm{e}-02$ \\
\hline 6 & 86121164 & $5.974250 \mathrm{e}-09$ & $3.523677 \mathrm{e}-01$ & 1.0000000000 & $1.000000 e+00$ \\
\hline 7 & 100156345 & $3.847627 \mathrm{e}-09$ & $7.956708 \mathrm{e}-01$ & 1.0000000000 & $1.000000 \mathrm{e}+00$ \\
\hline 8 & 110498058 & $1.615670 \mathrm{e}-08$ & $4.252635 \mathrm{e}-06$ & 0.0001627750 & $8.930533 e-05$ \\
\hline 9 & 120101077 & 2.428462e-09 & $9.607737 e-01$ & 1.0000000000 & $1.000000 \mathrm{e}+00$ \\
\hline 10 & 143000584 & $7.288953 \mathrm{e}-09$ & $1.483730 \mathrm{e}-01$ & 1.0000000000 & $1.000000 \mathrm{e}+00$ \\
\hline 11 & 147432746 & $9.236977 \mathrm{e}-09$ & $2.559803 e-02$ & 0.2177326427 & $3.327743 e-01$ \\
\hline 12 & 153096065 & $9.539061 \mathrm{e}-09$ & $1.874136 \mathrm{e}-02$ & 0.1793373396 & $2.623791 \mathrm{e}-01$ \\
\hline 13 & 164915166 & $5.593989 \mathrm{e}-09$ & $4.302561 \mathrm{e}-01$ & 1.0000000000 & $1.000000 \mathrm{e}+00$ \\
\hline 14 & 175995572 & $2.424688 \mathrm{e}-09$ & $9.611238 \mathrm{e}-01$ & 1.0000000000 & $1.000000 \mathrm{e}+00$ \\
\hline 15 & 179935272 & $2.416950 \mathrm{e}-09$ & $9.619106 \mathrm{e}-01$ & 1.0000000000 & $1.000000 \mathrm{e}+00$ \\
\hline 16 & 188799598 & $7.036906 \mathrm{e}-09$ & $1.785710 \mathrm{e}-01$ & 1.0000000000 & $1.000000 \mathrm{e}+00$ \\
\hline 17 & 196432767 & $1.483696 \mathrm{e}-08$ & $1.559299 \mathrm{e}-05$ & 0.0002984208 & $2.806739 \mathrm{e}-04$ \\
\hline 18 & 203573473 & $1.574496 \mathrm{e}-08$ & $7.087725 \mathrm{e}-06$ & 0.0001808611 & $1.346668 \mathrm{e}-04$ \\
\hline 19 & 221794586 & $3.942981 \mathrm{e}-09$ & 7.788247e-01 & 1.0000000000 & $1.000000 \mathrm{e}+00$ \\
\hline 20 & 231397605 & $1.148519 \mathrm{e}-08$ & $1.859819 \mathrm{e}-03$ & 0.0203391219 & $2.789728 \mathrm{e}-02$ \\
\hline 21 & 242970474 & $6.522862 \mathrm{e}-09$ & $2.540340 \mathrm{e}-01$ & 1.0000000000 & $1.000000 \mathrm{e}+00$ \\
\hline
\end{tabular}

Table 1: Estimation of the peaks and p-values corrections for multiple testing (Beniamini-Hochberg, Holm).

to examine in terms of expression and properties the corresponding genomic areas.

\section{Final Remarks}

The goal of this contribution was to provide some statistical considerations on the real nature of a hotspot. Statistical criteria for the identification of regions which are favoured by integrations (CIS or hotspots) are needed. We approached this problem by considering CIS not just like an area with very close integrations but like an area with very high integration density. Thus, we provide the null hypothesis based on kernel estimation of the P-H distribution, when integration are uniformly distributed over the genome. This criteria can be extended by considering a rectangular kernel, which better resemble the finite support of the integration distribution. Moreover, based on the proposed criteria, we can com- pare the "P-H hotspot" with the transcription start site distribution to distinguish which "P-H hotspot" reflects the high gene density areas, and which can really be thought as a real "hotspot" and thus leading to genetic investigations.

\section{Aknowledgements}

We greatly aknowledge Barbara Cassani, Alessandro Aiuti and Fulvio Mavilio for helping in understanding the crucial aspects of data and biological issue.

\section{References}

1. Ambrosi A, Cattoglio C, di Serio C (2008) Retroviral Integration Process in the Human Genome: Is It Really Non-Random? A New Statistical Approach. PLoS Comput Biology Vol. 4, Issue 8 / e1000144. „ CrossRef » Pubmed » Google Scholar 
2. Abel U, Deichmann A, Bartholomae C, Schwarzawaelder K, Glimm H, et al. (2007) Real-time definition of non-randomness in the distribution of genomic events. PLoS ONE 2: e570. » CrossRef » Pubmed » Google Scholar

3. Aiuti A, Ambrosi A, di Serio C, Bordignon et al. (2007) Multilineage hematopoietic reconstitution without clonal selection in ADA-SCID patients treated with stem cell gene therapy. Journal of Clinical investigation in press. " CrossRef » Pubmed » Google Scholar

4. Bushman F, Lewinski M, Ciuffi A, Barr S, Leipzig J, et al. (2005) Genomewide analysis of retroviral DNA integration. Nat Rev Microbiol 3: 848-858. » Pubmed » Google Scholar

5. Cassani B, Andolfi G, di Serio C, Ambrosi A, et al. (2006) Clonal analyses of retroviral vector integrations in ADASCID patients treated with stem cell gene therapy. Blood 108: 927A-927A 3249.

6. Cattoglio C, Facchini G, Sartori D, Antonelli A, Miccio A, et al. (2007) Hot spots of retroviral integration in human CD34(+) hematopoietic cells. Blood 110: 1770-1778. »CrossRef » Pubmed » Google Scholar

7. Hastie TJ, Tibshirani RJ (1990) Generalized Additive Models, New York: Chapman and Hall.» CrossRef» Google Scholar

8. Hematti P, Hong B, Ferguson C, Adler R, Hanawa H, et al. (2004) Distinct Genomic Integration of MLV and SIV

Research Article JCSB/Vol.2 January-February 2009

Vectors in Primate Hematopoietic Stem and Progeni PLoS Biol 2: 2183-2190. » CrossRef » Pubmed » Google Scholar

9. Jeroen R, Anthony U, Jaap K, Marcel R, Lodewyk W (2006) Detecting Statistically Significant Common Insertion Sites in Retroviral Insertional Mutagenesis Screens. PLoS Computational Biology Volume 2, Issue 12. " CrossRef » Pubmed " Google Scholar

10. Rick S, Mitchell, Brett FB, Astrid RW, Schroder2, et al. (2004) Retroviral DNA Integration: ASLV, HIV, and MLV Show Distinct Target Site Preferences. PLoS Biology Volume 2 | Issue 8 | e234. ” CrossRef » Pubmed » Google Scholar

11. Montini E, Cesana D, Ambrosi A, di Serio C, Naldini L, et al. (2006) Hematopoietic stem cell gene transfer in a tumor prone mouse model uncovers low genotoxicity of lentiviral vector integration. Nature Biotec 24: 687-96. » CrossRef » Pubmed " Google Scholar

12. Recchia A, Mavilio F, et al. (2006) Retroviral vector integration deregulates gene expression but has no consequence on the biology and function of transplanted $\mathrm{T}$ cells. PNAS 103: 1457-1462. " CrossRef » Pubmed » Google Scholar

13. Suzuki T, Shen H, Akagi K, Morse HC, Malley JD, et al. (2002) New genes involved in cancer identified by retroviral tagging. Nat Genet 32: 166-174. " " Pubmed » Google Scholar 